\title{
Increase in intracellular proline content in Anabaena variabilis during stress conditions
}

\author{
M ayashree B Syiem* and Natasha A Nongr um \\ Department of Biochemistry, North Eastern Hill University, Shillong-793 022, Meghalaya, INDIA \\ *Corresponding author. E-mail: mayashreesyiem@yahoo.co.in
}

\begin{abstract}
The present study deals with the effects of stress due to varying concentrations of sodium chloride, cadmium, $\mathrm{pH}$ and exposure to pesticides on intracellular proline accumulation in Anabaena variabilis in laboratory conditions. The stresses induced due to these changes in their growth media were expressed as decrease in growth (by $40 \%$ at pH 5; $30 \%$ at $50 \mathrm{mM} \mathrm{NaCl} ; 80 \%$ at $50 \mu \mathrm{M}$ endosulphan and $55 \%$ at $10 \mu \mathrm{M} \mathrm{CdSO}$ ) as measured in terms of chlorophyll a concentrations in the test organism. Low pH $(5,6)$ had stronger negative effect on growth than increasing alkaline $\mathrm{pH}$. The cyanobacterium showed varying degree of susceptibility to increasing salt, cadmium and endosulphan concentrations. Under all these altered conditions, their intracellular proline concentration was found to increase linearly in the test organism with increase in its amount of stress inducing substances in its vicinity. Proline appears to be a stress provoked substance in A. variabilis. However, there seemed to be a breakdown in the proline synthesis mechanism when concentrations of such compounds reached toxic proportions.
\end{abstract}

Keywords: Cyanobacteria, $\mathrm{NaCl}$, Cadmium, pH, Endosulphan, Intracellular proline

\section{INTRODUCTION}

Microbes in their natural environment are constantly challenged by various stresses in diverse forms viz changes in temperature, moisture (droughts and floods), salinity and so forth. Salinity is now known to be involved in remarkably reducing the growth and productivity of plants, eukaryotic microorganisms and bacteria (Inabha et al. 2001) and affects agricultural productivity in areas with saline soils. In addition, manmade pollutants further add to the stresses encountered by these organisms. Increasing industrial activities release heavy metals in to the environment which cannot be further transmuted to totally innocuous forms. Many heavy metals are readily taken up by crops and fruits, which subsequently results in movement through the food chain and their ultimate accumulation in the highest trophic level in humans. One such heavy metal contaminant is cadmium $(\mathrm{Cd})$. Agricultural soils are mainly contaminated with $\mathrm{Cd}$ from the extensive use of fertilizers, dispersal of sewage sludge and atmospheric deposition. $\mathrm{Cd}$ accumulates in the body replacing zinc in the liver and kidneys leading to serious diseases. It is also known for its phytotoxicity to germinating rice seeds resulting in adverse effects on RNA levels, ribonuclease activity, phosphorolytic and proteolytic enzymes, and thus compromising productivity (Shah and Dubey, 1998).

Soil is a dynamic system in which the physical, chemical and biotic components are in a state of equilibrium. Application of insecticides such as endosulphan without taking care of the other soil constituents disturbs this equilibrium which adversely affects the productivity of the soil. Pesticides reaching the soil affect non-target organisms and their activities severely limiting beneficial soil microorganisms such as cyanobacteria (Abdel-Raouf and El-Shafey, 2009).

Modern agriculture finds increasing use of herbicides and pesticides in crop cultivation in a bid to enhance yield. Even though cyanobacteria are not the target organisms, application of insecticides (many of which interfere with the process of photosynthesis) has adverse effects on them as they are actively engaged in carbon fixation via photosynthesis. However, cyanobacteria are resilient organisms and combat such stresses in their environment with elaborate protective mechanisms to ensure their survival. Previous researchers have shown proline accumulation in plants, bacteria, protozoa, algae and marine invertebrates in response to stress in their surroundings (Delauney and Verma, 1993; Fatma et al., 2007).

Proline has been known as important indicator for stress tolerance and functions as stabilizer (Shah and Dubey, 1998), a metal chelator (Farago and Mullen, 1979), as an inhibitor of lipid peroxidation (Mehta and Gaur, 1999), a hydroxyl radical (Smirnoff and Cumbes, 1989), and a singlet oxygen scavenger (Alia et al., 2001). Not many reports are available on role of proline in stress tolerance in cyanobacteria except for a report on impact of environmental pollution on cyanobacterium Westiellopsis prolofica by Fatma et al. (2007). 
One of the potential biotechnological applications of cyanobacteria is their role as bioremediator in polluted aquatic systems. However, polluted environments present stress to the cyanobacteria which prompts the organisms to develop protective strategies. The aim of the present study was to find out the accumulation of intracellular proline in cells of Anabaena variabilis and its role when presented with stress in their surroundings.

\section{MATERIALS AND METHODS}

Soil samples were collected from various rice fields during the month of May. For isolation of cyanobacteria, $1 \mathrm{~g}$ soil was vortexed in $99 \mathrm{ml}$ of sterile water (Jurgensen and Davey, 1968). The mixture was used to inoculate Petridishes containing $2.5 \%$ nutrient agar made in BG 11 medium (Rippka et al., 1979). All Petri-dishes were incubated on a 14 / $10 \mathrm{~h}$ light / dark cycle with a light intensity of $50 \mu \mathrm{mol} \mathrm{m}{ }^{-2} \mathrm{sec}^{-1}$ at $25 \pm 2^{\circ} \mathrm{C}$. Individual colonies developed were picked up and transferred to liquid BG $11_{0}$ medium and further purified by repeated pour plate method. The cyanobacterium Anabaena variabilis was selected for the study as this strain was isolated from almost all of the fields undertaken for study and thus is perceived as widely present in most rice field ecosystems. Cultures were maintained in BG 11 medium. For the experiments, axenic Anabaena variabilis was grown in $\mathrm{BG} 11_{0}$ medium with varying $\mathrm{pH}(5,6,7,8$ and 9); $\mathrm{NaCl}$ concentrations $(0,50,100,200,300,400$ and 500 $\mathrm{mM}) ; \mathrm{CdSO}_{4}$ concentration $(0,5,10,15,20,25,30$ and 40 $\mu \mathrm{M})$ and endosulphan concentration $(5,10,20,30,40,50$, $60 \mu \mathrm{M})$. The cyanobacterial biomass was harvested on eighth day after inoculation by centrifuging at $10,000 \mathrm{~g}$ for $5 \mathrm{~min}$. The pellets obtained were used for estimating chlorophyll a (Mackinney, 1941) and proline content (Bates et al. 1973) using a UV-VISIBLE spectrophotometer (Variance Cary 50 Bio).

\section{RESULTS AND DISCUSSION}

Relation between increase in intracellular proline and heavy metal stress in plants has been reported by many researchers (Alia and Saradhi, 1991; Hanson and Burnet, 1994; Mansour et al. 2005). The role of proline has also been indicated in adjusting osmotic stress (Watanabe et al. 2000) and in reducing oxidative stress resulting from enhanced level of salinity in the vicinity (Hare et al. 1998). Not many reports have focussed their attention towards the role of intracellular proline in stress management in cyanobacteria. Fatma et al. (2007) have reported impact of environmental pollution on the cyanobacterium Westiellopsis prolofica where they have identified intracellular proline as one of the metabolites that helps alleviate stress in the experimental organism. Taking cue from their work the present study had investigated increase in intracellular proline content in the cyanobacterium $A$. variabilis in presence of frequently occurring undesirable elements in its vicinity in an attempt to further understand and establish proline as a common metabolite that comes into play during stress management in cyanobacteria.

The experimental organism was isolated from various rice fields of Meghalaya during a diversity study. Meghalaya presents unique geographical and climatic challenges to its faunal and floral population. Among these is acidic $\mathrm{pH}$ of soil and water that is often recorded in the rice fields of the state (Syiem et al., 2010). Salinity is another common stress experienced by these microbes. Other common unwanted compounds are herbicides and pesticides used during cultivation and heavy metals due to mining activities in the state. Thus, in this study changes in $\mathrm{pH}, \mathrm{NaCl}$, pesticide endosulphan and $\mathrm{CdSO}_{4}$ concentrations had been used as stresses on the cyanobacterium, A. variabilis.

A study of this organism in varying $\mathrm{pH}$ was intriguing as it was isolated from acidic rice fields. Cyanobacterial growth was susceptible to $\mathrm{pH}$ change in the medium. The effect was more pronounced in acidic $\mathrm{pH}(5,6)$ compared to cultures growing in higher $\mathrm{pH}(8,9)$. The growth of Anabaena variabilis was reduced by $26 \%$ in $\mathrm{pH} 6$ while a further drop in $\mathrm{pH}$ by another unit of $1(\mathrm{pH}$ 5) reduced the growth by $40 \%$ compared to the controlled cultures ( $\mathrm{pH}$ 7.6). However, in alkaline $\mathrm{pH}$ the reduction in growth was much milder in nature $(12 \%$ in $\mathrm{pH} 8$ and $14 \%$ in $\mathrm{pH} 9$ ). Intracellular proline content increased in all experiments when $\mathrm{pH}$ deviated from the ideal value of 7.6 (Fig. 1). This increase in proline content was comparable in all range of $\mathrm{pH}$ change even though growth was visibly compromised in $\mathrm{pH} 5$ and 6 . Further on comparison, proline accumulation was not found to be proportional to the changes in chlorophyll a content brought about by variation in $\mathrm{pH}$ values of the media. Thus, defence mounted by the cyanobacterium in form of increased levels of intracellular proline may not distinguish the differential severity of the adverse condition that arises because of change in hydrogen ion concentration of the growth media.

Reduced photosynthetic activity and growth has earlier been reported in cyanobacteria due to increase in salinity (Schubert et al. 1993). $50 \mathrm{mM} \mathrm{NaCl}$ in the growth medium reduced chlorophyll a content by about $30 \%$ (Fig 2). Beyond that the decline was gradual with increasing $\mathrm{NaCl}$ concentration up to $400 \mathrm{mM}$. The decrease in chlorophyll a content was only $18 \%$ between $50 \mathrm{mM}$ and $400 \mathrm{mM}$ concentration of $\mathrm{NaCl}$. But the percent decrease in chlorophyll a content was close to $30 \%$ between 400 and $500 \mathrm{mM}$. Intracellular proline in cells under $\mathrm{NaCl}$ stress recorded a gradual increase with increasing $\mathrm{NaCl}$ concentration up to $300 \mathrm{mM}(11-45 \%)$. However the increase in proline concentration was comparatively less in $\mathrm{NaCl}$ concentration beyond $300 \mathrm{mM}$ (Fig. 2). This may 


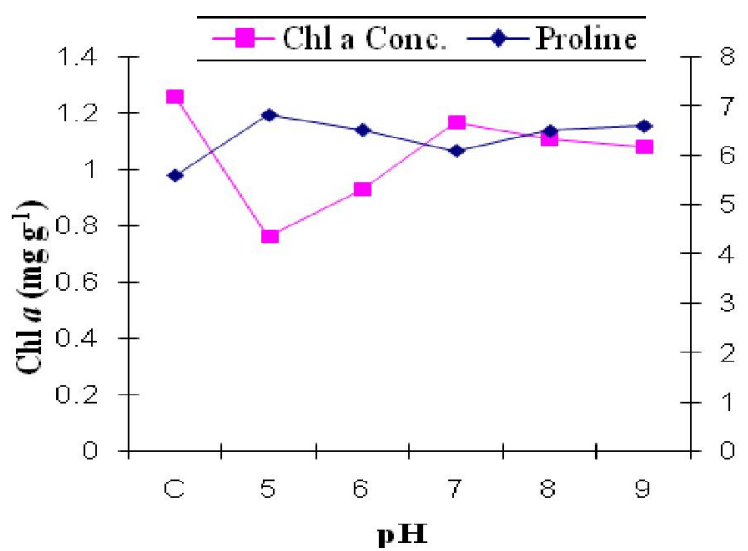

Fig. 1. Effect of varying $\mathrm{pH}$ on the chlorophyll $\mathrm{a}$ and proline content of Anabaena variabilis.

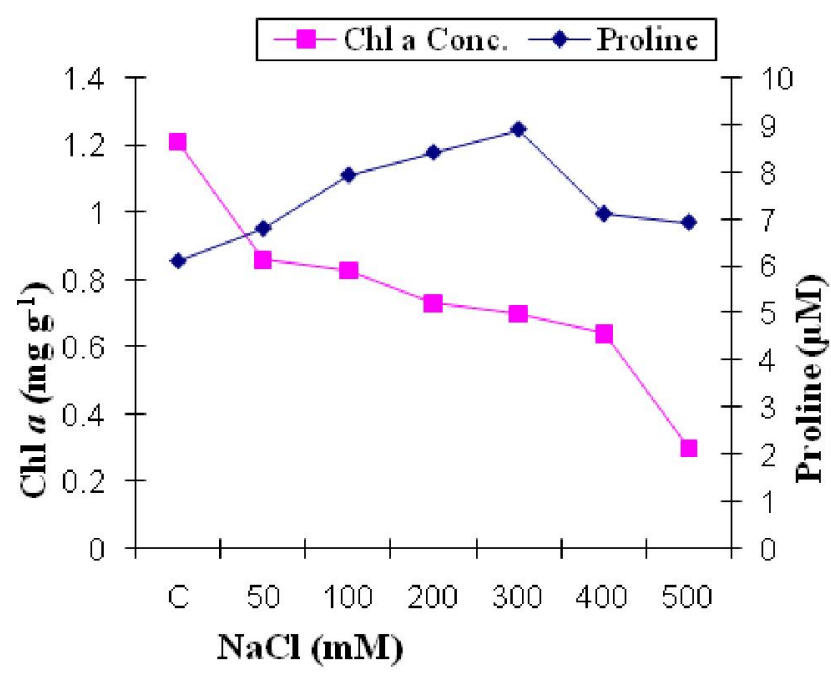

Fig: 2. Effect of different concentrations of $\mathrm{NaCl}$ on the chlorophyll a and proline content of Anabaena variabilis.

be due to the fact that at such high concentrations of sodium chloride, many of the vital metabolic activities are compromised by ionic imbalance that may also affect proline synthesis.

The pesticide endosulphan is often used in rice cultivation. However, effect of endosulphan application has not been studied in detail on the microbes that exist in the rice field ecosystem and are beneficial to crop plants and help maintain the soil quality and fertility. A study involving increasing concentrations of endosulphan in the vicinity of the test organism showed negative effect on their growth (Fig. 3). Micro molar concentrations of endosulphan in the medium could visibly reduce the growth in terms of chlorophyll a content.

A. variabilis could tolerate an increase up to $30 \mu \mathrm{M}$ endosulphan. This tolerance level could be attributed to sharp rise in intracellular proline in these organisms as the concentration of endosulphan increased in the medium. However as in the case with $\mathrm{NaCl}$, beyond a certain concentration of endosulphan, the cells showed severe drop in their capacity to synthesize proline (Fig. $3)$. The fall in proline synthesis is in tune with decline in their growth. Chlorophyll a content was only $\sim 20$ and $10 \%$ of that of control culture in media that contained 50 and $60 \mu \mathrm{M}$ concentration of endosulphan. Proline concentration had reached a value $\sim 250 \%$ of that of control in presence of $20 \mu \mathrm{M}$ endosulphan in the medium, where growth was recorded to be $83 \%$ of the control. However proline synthesis seems to have been affected by increased concentration of endosulphan in the medium. This may be the cause of visible decline seen in growth as intracellular proline was not made in sufficient amount to provide protection against endosulphan toxicity. The proline concentration has fallen by $20 \%$ below control value in medium containing $60 \mu \mathrm{M}$ endosulphan.

Exposure to heavy metals is known to affect cyanobacterial growth (Leena and Fatma, 2000; Fatma et al., 2007). Metal stress in form of increasing concentration of $\mathrm{CdSO}_{4}$ was also expressed as reduction in Chlorophyll a content. At $10 \mu \mathrm{M} \mathrm{CdSO}_{4}$ in the medium, chlorophyll a concentration declined by $55 \%$ which further went down to $98 \%$ in presence of $35 \mu \mathrm{MCdSO}_{4}$ (Fig. 4). The effect of $\mathrm{CdSO}_{4}$ was most severe among the four different stresses tested on the cyanobacterium. Again, a rise in intracellular proline content was noted with increasing concentration of $\mathrm{CdSO}_{4}$ in the growth medium that decline as concentration reached higher values of $30 \mu \mathrm{M}$ and above. (Fig.4). In other words, as adverse effect was expressed as reduction in chlorophyll a concentration, increase in intracellular proline synthesis with rise in unwanted compounds in the vicinity could be looked at as the cyanobacterium's strategy to combat such undesirable conditions. However at higher toxicity levels even proline synthesis itself seemed to be compromised. As we compare the different stresses presented to the cyanobacterium A. variabilis, the toxic effect on chlorophyll a concentration was in the order of $\mathrm{CdSO}_{4}>$

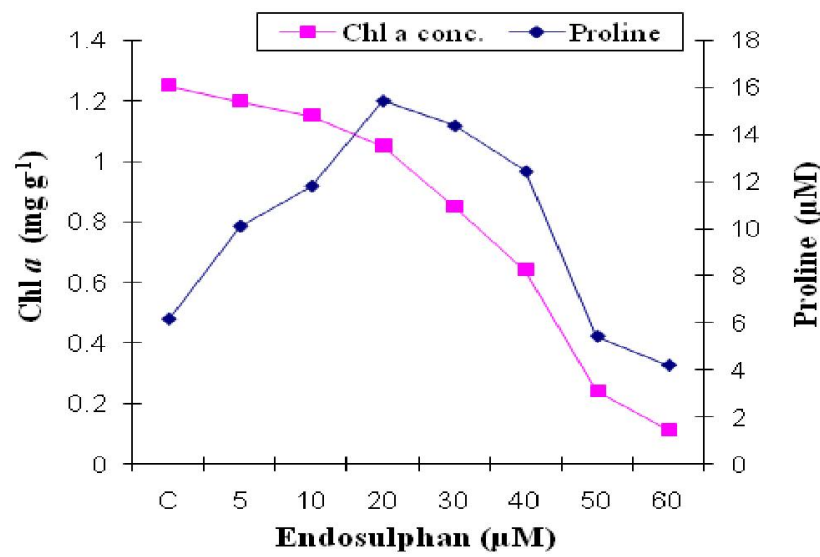

Fig. 3. Effect of different concentrations of endosulphan on the chlorophyll a and proline content of Anabaena variabilis. 


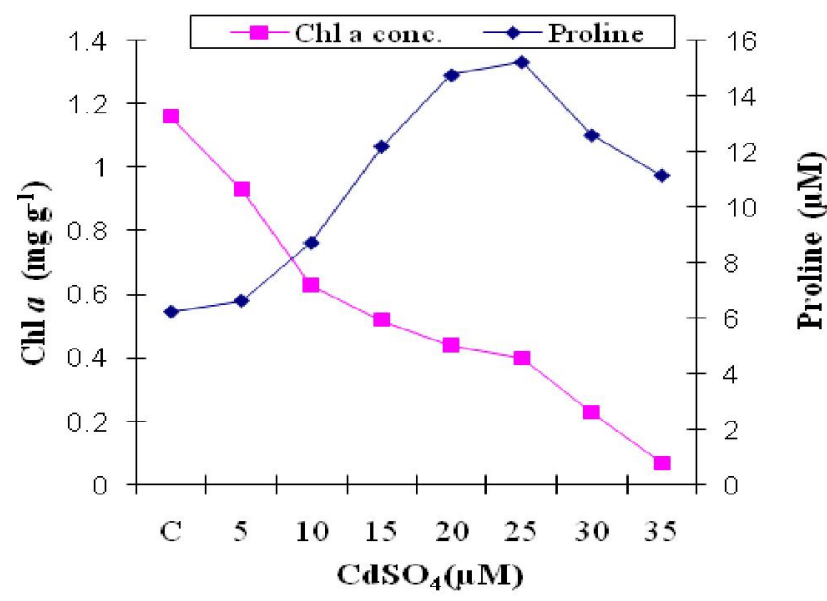

Fig. 4. Effect of different concentrations of $C$ admium sul phate on the chlorophyll a and proline content of Anabaena variabilis.

endosulphan $>\mathrm{NaCl}>$ changes in $\mathrm{pH}$. $\mathrm{CdSO}_{4}$ and the pesticide endosulphan seem to be more toxic as their toxicity was enunciated at micro molar concentrations. Endogenous proline accumulation was always enhanced whenever the cyanobacterium was subjected to any form of adversity in growth medium. There seems to be an inverse relationship between decrease in chlorophyll a content due to adverse environment and increase in intracellular proline accumulation in A. variabilis. Thus proline seems to be a stress induced metabolite in this cyanobacterium. However, beyond a certain concentration of stress compounds, the increase in proline content did not follow this linear relationship. It has been suggested at least in the case of heavy metal exposure that heavy metal ions bind to sulfahydryl groups of enzymes essential for catalysis that leads to death of cyanobacteria (Rosko and Rachlin, 1975). This may be the reason for reduced proline synthesis at toxic levels of these stress compounds (eg. in endosulphan concentration beyond $40 \mu \mathrm{M} ; \mathrm{CdSO}_{4}$ beyond $20 \mu \mathrm{M}$ and $\mathrm{NaCl}$ beyond $300 \mathrm{mM}$ ). Increased in intracellular proline concentration was highest in presence of endosulphan. An increase of $147 \%$ was recorded in presence of $20 \mu \mathrm{M}$ endosulphan in the growth medium. The order of increase in intracellular proline accumulation was $20 \mu \mathrm{M}$ endosulphan $>25 \mu \mathrm{M} \mathrm{CdSO}_{4}>300 \mathrm{mM} \mathrm{NaCl}>\mathrm{pH} 5$.

\section{ACKNOWLEDGEMENTS}

The authors would like to thank UGC, New Delhi for financial support received under Rajiv Gandhi national fellowship and University with potentials for excellence scheme extended to School of Life Sciences, North Eastern Hill University, Shillong, Meghalaya.

\section{REFERENCES}

Abdel-Raouf, N. and El-Shafey, N.M. (2009). Harmful effects of endosulfan treatment on cyanobacterial distribution and some macromolecules of soybean plant. African J ournal of
Biotechnology, 8 (22): 6277-6281.

Alia and Saradhi, P. P. (1991). Proline accumulation under heavy metal stress. J Plant Physiol., 138:554-558.

Alia, Mohanty, P. and Matysik, J. (2001). Effect of Proline on the production of singlet oxygen. Amino Acid, 21: 195-200.

Bates, L.S., Waldren, R.P. and Teare, I. D. (1973). Rapid determination of free proline for water stress studies. Plant Soil, 39: 205-207.

Delauney, A. and Verma, D.P.S. (1993). Proline biosynthesis and osmoregulation in plants. Plant J., 4: 215-223.

Farago, M.E. and Mullen, W.A. (1979). Plants which accumulate metals. Part IV. A possible copper-Proline complex from the roots of Armeria maritima. Inorg C him Acta, 32: 93-94.

Fatma, T., Khan, M.A. and Choudhary, M. (2007). Impact of environmental pollution on cyanobacterial proline content. J Appl Phycol., 19: 625-629.

Hanson, A.D. and Burnet, M. (1994). Evolution and metabolic engineering of osmoprotectant accumulation in higher plants. In: Cherry JH (ed) Biochemical and cellular mechanisms of stress tolerance in plants. Springer, Berlin, 291-302.

Hare, P.D., Cress, W.A. and Van Staden, J. (1998). Dissecting the roles of osmolyte accumulation during stress. Plant C ell Environ, 21:535-553.

Inabha, M., Sakamato, A. and Murata, N. (2001). Functional expression in E.coli of low affinity and high affinity $\mathrm{Na}^{+}$ $(\mathrm{Li})^{+} / \mathrm{H}^{+}$antiporters of Synechocystis. J. Bacteriol., 183: 1376-1384.

Jurgensen, M.F. and Davey, C.B. (1968). Nitrogen fixating blue-green algae in acid forest and nursery soils. Can. J. Microbiol., 14: 1179.

Leena, T. and Fatma, T. (2000). Potential use of Phormidium for bioremediation of copper. Ind. J. Appl. Pure Biol., 15: 83-86.

Mackinney, G. (1941). Absorption of light by chlorophyll solutions. J.Biol.Chem., 140: 315-322.

Mansour, M.M.F., Salama, K.H.A., Ali, F.Z.M. and Abou Hadid, A.F. (2005). Cell and plant responses to $\mathrm{NaCl}$ in Zea mays L. Cultivars differing in salt tolerance. G en Appl Plant Physiol., 31:29-41.

Mehta, S. and Gaur, J.P. (1999). Heavy metal induced proline accumulation and its role in ameliorating metal toxicity in chlorella vulgaris. New Phytol., 143: 253-259.

Rippka, R., Dervelles, J., Waterbury, J.B., Herdman, M. and Stanier, R.Y. (1979). Genetic assignment, strain histories and properties of pure culture of cyanobacteria. J.Gen. Microbiol., 111: 1-61.

Rosko, J. J. and Rachlin, J. W. (1975). The effect of copper, zinc, cobalt and manganese on the growth of marine diatom Nitzchia closterium. Bull Torr Bot Club. 102:100-106.

Schubert, H., Fulda, S. and Hagemann, M. (1993). Effects of adaptation to different salt concentrations on photosynthesis and pigmentation of the cyanobacterium Synechocystis sp. PCC 6083.J Plant Physiol., 142:291-295.

Shah, K. and Dubey, R.S. (1998). Effect of Cadmium elevates the protein level and alters the activity of proteolytic enzymes in germinating rice seeds. Acta Physiologiae Plantarum., 20: 189-196.

Smirnoff, N. and Cumbes, Q.J. (1989). Hydroxyl radical scavenging activity of compatible solute. Phytochemistry, 28: 1057-1060. 
Syiem, M.B., Nongbri, B.B., Pinokiyo, A., Bhattacharjee, A., Nongrum, N.A. and Hynniewta, L. (2010). Significance of cyanobacterial diversity in different ecological conditions of Meghalaya, India. J . Appl. \& N at. Sci., 2(1):134-139.
Watanabe, S., Kojima, K., Ide, Y., and Sasaki, S. (2000). Effects of saline and osmotic stress on proline and sugar accumulation in Populus euphratica in vitro. Plant C ell Tissue Organ Cult., 63:199-206. 